\section{Baixo peso ao nascer em adolescentes e adultas jovens na Região Nordeste do Brasil}

André Henrique do Vale de Almeida 1 Maria Conceição Oliveira Costa 2 Silvana Granado Nogueira da Gama 3 Magali Teresópolis Reis Amaral 4 Graciete Oliveira Vieira 5

\section{Low weight at birth in adolescent and young adult mothers in the Northeast Region of Brazil}

\section{Abstract}

Objectives: to evaluate the association between characteristics relating to socio-demographic factors and the prenatal and delivery period of adolescent and young adult mothers whose newborns present with low weight at birth in State capitals and other municipalities in the Northeast region of Brazil, between 2011 and 2012.

Methods: a hospital-based cross-sectional study was carried out at regional level in 2011 and 2012 Information was obtained by way of an interview with the mother immediately post-partum and from data contained in the medical records of mothers and newborns. A conceptual theoretical model was established with three hierarchical levels and the dependent variable was low weight at birth (LWB).

Results: of the 3009 mothers interviewed, 9.7\% had had newborns of low birth weight. The mothers who had received less than six prenatal consultations had a 1.7 times greater chance of giving birth to a child of low weight; a first pregnancy increased the risk of occurrence of $L W B$ by an order of 1.5; while prematurity was the principal exposure factor, with 21 greater likelihood of this outcome.

Conclusions: the study showed a high frequency of low birth weight among adolescent and young adult mothers, the main associated factors being an insufficient number of prenatal consultations, a first pregnancy and prematurity.

Key words Teenage pregnancy, newborns low weight, Adolescent, Young adult

\section{Resumo}

Objetivos: avaliar a associação entre as características sociodemográficas, do pré-natal e do parto de mães adolescentes e adultas jovens com o baixo peso ao nascer, em capitais e outros municipios da Região Nordeste do Brasil, no período de 2011 e 2012.

Métodos: estudo transversal, de base hospitalar e nivel regional, realizado em 2011 e 2012. As informações foram obtidas por meio de entrevista com a puérpera no pós-parto imediato e coletados dados dos prontuários maternos e dos recém-nascidos. Um modelo teórico conceitual foi estabelecido com três niveis de hierarquia e a variável dependente foi o baixo peso ao nascer (BPN).

Resultados: do total de 3009 puérperas entrevistadas, 9,7\% apresentaram conceptos com baixo peso. As puérperas que realizaram menos que seis consultas no pré-natal apresentaram uma chance 1,7 vezes maior de ter $R N$ com baixo peso; ser primigesta aumentou a chance da ocorrência do BPN em 1,5 vezes; assim como a prematuridade que representou o principal fator de exposição, elevando em 21 vezes a chance desse desfecho.

Conclusões: o estudo demonstrou alta frequência de baixo peso ao nascer entre mães adolescentes e adultas jovens, tendo como principais fatores associados o número insuficiente de consultas pré-natal, primigestação e prematuridade.

Palavras-chave Gravidez na adolescência, Recémnascido de baixo peso, Adolescente, Adulto jovem 


\section{Introdução}

A gravidez na adolescência é um evento globalmente estudado, com diferenças significativas entre os distintos contextos e regiões do mundo, que tem suscitado interesse e preocupação, principalmente nos países subdesenvolvidos. ${ }^{1}$ No Brasil, a sua ocorrência não é um achado raro, sendo que uma em cada cinco brasileiras dá à luz ao primeiro filho antes dos 20 anos de idade. ${ }^{2}$ Apesar da queda na taxa de fecundidade geral no Brasil, os indicadores de 2010 apontaram que os partos entre adolescentes representaram cerca de $20 \%$ do total de nascimentos, chegando a $22 \%$ na Região Nordeste. ${ }^{3}$

$\mathrm{O}$ baixo peso ao nascer (BPN) é um indicador de saúde essencialmente relevante entre os fatores associados à morbimortalidade perinatal.4 A prematuridade e o crescimento intrauterino restrito (CIUR) são os dois fatores que, associados ou isolados, resultam em nascimentos de recém-nascidos com este desfecho. 5

O BPN é considerado o fator de risco isolado de maior magnitude para a mortalidade infantil. No nível mundial, a mortalidade neonatal é 20 vezes mais provável para recém-nascidos (RN) com baixo peso, em comparação com os de peso adequado ao nascer. ${ }^{6}$ Nesta perspectiva, mães adolescentes apresentam as maiores taxas, entretanto, observa-se maior percentual de primíparas nesse grupo, sugerindo que fatores biológicos ligados à imaturidade podem estar associados à maior ocorrência desse desfecho. 7,8

Nos diferentes contextos, o coeficiente de BPN varia com importante desvantagem para os países menos desenvolvidos, devido às condições socioeconômicas desfavoráveis, sendo considerado como um relevante indicador de saúde da população e importante marcador de desenvolvimento social. ${ }^{9}$ No Brasil, tem se observado taxas mais elevadas nas Regiões mais desenvolvidas (Sul e Sudeste), comparadas às Regiões menos desenvolvidas (Norte e Nordeste), configurando o chamado paradoxo epidemiológico do baixo peso, levando especialistas a dedicarem estudos que busquem explicação para esse paradoxo. 10

A despeito desses achados, estudos realizados em outros países, em maternidades de alto nível socioeconômico, onde são minimizados os efeitos de uma assistência ao pré-natal e ao nascimento inadequadas, demonstram resultados perinatais semelhantes entre adolescentes e mulheres de faixas etárias mais avançadas, o que parece minimizar os efeitos atribuídos à idade materna, deslocando tais efeitos para o campo dos fatores sociais e econômicos. 11,12 Assim, a identificação dos principais fatores de risco que deixam vulneráveis os filhos de mães adolescentes e adultas jovens é de fundamental importância, para subsidiar programas e ações que contribuam na redução das taxas de BPN, especialmente em regiões mais carentes, como a Região Nordeste.

Considerando-se a relevância de pesquisas que utilizam dados representativos regionais no contexto de um país com dimensão continental, este artigo tem como objetivo avaliar a associação entre as características sociodemográficas, do pré-natal e do parto de mães adolescentes e adultas jovens com o baixo peso ao nascer, em municípios da Região Nordeste do Brasil.

\section{Métodos}

Esta pesquisa representa um subprojeto que faz parte do inquérito nacional sobre o parto e o nascimento, pesquisa Nascer no Brasil.13 Estudo epidemiológico transversal de base hospitalar, realizado no período de fevereiro de 2011 a outubro de 2012, cujo objetivo foi analisar as condições de assistência à gestação, ao parto e seus principais desfechos em nível nacional.

Para compor a amostra foram elegíveis as instituições de saúde que registraram 500 partos/ano ou mais segundo o Sinasc, em 2007. Os sujeitos do estudo foram mulheres admitidas nas maternidades selecionadas por ocasião do parto e seus conceptos vivos, independente da idade gestacional ou peso, ou mortos com idade gestacional $\geq 22$ semanas de gestação ou peso ao nascer $\geq 500 \mathrm{~g}$. Foram excluídas as puérperas cujo parto tivesse ocorrido no domicílio, no transporte para a maternidade ou em outra unidade de saúde não selecionada.

Para a pesquisa nacional, realizada em todas as regiões do país, representadas pelos municípios selecionados, o tamanho da amostra em cada estrato foi calculado com base na taxa geral de cesariana em 2007 (46,6\%), para detectar diferenças de $14 \%$ entre os tipos de serviço, considerando nível de significância de $5 \%$, poder de estudo de $95 \%$ e efeito desenho de 1,3, totalizando um tamanho amostral de 450 mulheres por estrato.

A amostra nacional foi selecionada em três estágios. No primeiro, os hospitais elegíveis foram estratificados pelas cinco macrorregiões do país (Norte, Nordeste, Sul, Sudeste e Centro-Oeste), por localização (capitais e outros municípios) e por tipo de serviço (público, privado e misto), com probabilidade de seleção proporcional ao número de partos em 2007, em cada um dos estratos. No segundo 
estágio, um método de amostragem inversa foi utilizado para selecionar o número de dias necessários para entrevistar 90 puérperas em cada hospital, tendo o trabalho de campo a duração mínima de sete dias. No terceiro estágio, foram selecionadas as mulheres elegíveis a cada dia do trabalho de campo.

A pesquisa foi realizada em 191 municípios de todos os Estados e Regiões do Brasil, sendo 27 capitais e 164 municípios do interior. No que diz respeito à Região Nordeste, foram incluídas 68 unidades de saúde distribuídas em 54 municípios. O desenho da amostra em detalhes está publicado em artigo científico. ${ }^{14}$

A coleta de dados foi realizada por equipes de estudantes e profissionais de saúde, treinados e supervisionados pela coordenação da pesquisa, utilizando questionário eletrônico com auxílio de notebooks. Para o presente estudo foram analisados apenas os dados relacionados à amostra da região Nordeste, capitais e municípios do interior, que inclui as entrevistas hospitalares com as adolescentes e adultas jovens no pós-parto imediato, além de coleta de dados maternos e do recém-nascido nos prontuários das maternidades selecionadas, considerando-se elegíveis mães cujo resultado gestacional tenha sido feto único.

Foram consideradas como adolescentes as puérperas com idade de 10 a 20 anos incompletos, e adultas jovens aquelas entre 20 a 24 anos. As mulheres que se declararam amarelas ou indígenas foram excluídas, devido à pequena proporção na população.

Baseado na literatura foi elaborado um modelo teórico conceitual com três níveis de hierarquia (distal; intermediário e proximal). No nível distal, foram incluídas como variáveis os aspectos socioeconômicos: residência (interior e capital); estado civil (sem companheiro e com companheiro); faixa etária ( 10 a 19 anos e 20 a 24 anos); adequação da escolaridade com a idade (inadequada e adequada); ocupação remunerada (sim e não); e cor da pele (preta; parda e branca).

No nível intermediário, características obstétricas e de pré-natal: número de consultas no pré-natal ( $<6$ e $\geq 6$ consultas); primigestação; fumo durante a gestação; consumo de bebida alcoólica na gestação; e acompanhamento pelo mesmo profissional no pré-natal. No nível proximal, fatores relacionados às características obstétricas: financiamento para o parto, idade gestacional e tipo de parto. A variável dependente foi o BPN, definido como peso ao nascer menor que 2500 gramas. Para o cálculo da variável "adequação da escolaridade para a idade" foi consi-derado o número de anos de estudo esperado para a idade, sendo preconizado como padrão adequado ter concluído o ensino médio, e para a variável prematuridade foi utilizado um algoritmo construído para o cálculo da idade gestacional. 15

Para a presente pesquisa, inicialmente foi realizada análise bivariada, por meio do teste do quiquadrado de Person, para verificar associação entre o BPN e demais variáveis. Para a construção do modelo multivariado hierarquizado foram selecionadas as variáveis que apresentaram nível de significância $<0,20$, pelo teste de Wald. As variáveis foram organizadas por nível de proximidade com o desfecho, inserindo-se primeiro as do nível distal. As variáveis significativas $(p<0,05)$ foram conservadas no modelo e entraram no ajuste do nível intermediário. O mesmo procedimento foi empregado até que as variáveis proximais fossem ajustadas para as distais e intermediárias, sendo que as variáveis selecionadas pelo nível de significância estatística em um determinado nível permaneceram nos modelos subsequentes, mesmo que a inclusão de variáveis hierarquicamente inferiores modificasse sua significância. Os resultados foram expressos em valores de razão de chances, com seus respectivos intervalos de $95 \%$ de confiança.

Utilizou-se o programa SPSS versão 17.0 (Statistical Package for Social Science for Windows), e o módulo Complex Sample para correção do efeito do desenho, por se tratar de uma amostra complexa.

Este estudo foi submetido e aprovado pelo Comitê de Ética para Pesquisa da Universidade Estadual de Feira de Santana-BA. As puérperas entrevistadas e os diretores das instituições em que o trabalho foi realizado assinaram o termo de consentimento livre e esclarecido.

\section{Resultados}

Do total de 3009 puérperas entrevistadas nas capitais e demais municípios da Região Nordeste, 9,7\% apresentaram conceptos com BPN. No tocante às características sociodemográficas, observou-se que, 2398 $(79,7 \%)$ das mulheres residiam em municípios do interior dos Estados, e a maioria (77,6\%) tinha companheiro. As adolescentes representaram $43,8 \%$ do total dessas puérperas. Quanto à adequação da escolaridade com a idade, $1806(60,9 \%)$ era inadequada; $2509(83,4 \%)$ não trabalhavam e 2208 $(73,4 \%)$ se declararam de cor parda. Segundo as análises, nesse bloco de variáveis, apenas a procedência e a idade da mãe estiveram associadas 
com o desfecho em estudo (BPN).

Em relação às características de pré-natal, $64,7 \%$ das entrevistadas realizou seis ou mais consultas; $57,9 \%$ era primigesta, e $90,7 \%$ afirmaram ter sido acompanhada pelo mesmo profissional durante o pré-natal. Entre as entrevistadas, 5,6\% e 15,4\% afirmaram ter fumado e consumido bebida alcoólica na gestação, respectivamente. Neste grupo de variáveis, o número de consultas no pré-natal e a primigestação mostraram associação estatística $(p<0,05)$ com o baixo peso do RN.

No último bloco de variáveis, relacionadas às características obstétricas, $2870(95,4 \%)$ das participantes tiveram seu parto realizado no setor público; $321(10,9 \%)$ deram à luz RN prematuros; 1231 $(40,9 \%)$ submeteram-se à cesariana. Foi notada associação significativa entre o BPN e a prematuridade (Tabela 1).

A Tabela 2 mostra a análise de regressão logística, utilizando-se o modelo hierarquizado. No bloco distal (Modelo I) apresentaram significância as variáveis "procedência" e "idade", que foram mantidas no modelo, sendo assim inseridas no nível intermediário (Modelo II). Nesta etapa da análise, o número de consultas no pré-natal e a primigestação apresentaram associação, sendo que a procedência do interior e a faixa etária adolescente permaneceram significativas, esta última com resultado limítrofe (IC95\%: 1,0 - 1,6).

$\mathrm{Na}$ última etapa, (nível proximal - Modelo III) foram mantidos como principais fatores associados ao baixo peso: adolescentes e adultas jovens que realizaram menos do que seis consultas apresentaram chance cerca de duas vezes maior de dar à luz $\mathrm{RN}$ de baixo peso $(\mathrm{OR}=1,7)$; a primigestação, também contribuiu de forma significante para a sua ocorrência $(\mathrm{OR}=1,5)$; assim como a prematuridade que representou o principal fator de exposição, elevando em 22 vezes a chance $(\mathrm{OR}=21,8)$.

A procedência e a idade não mantiveram associação significante com o baixo peso ao nascer, após o ajuste com as variáveis dos níveis intermediário e proximal, tendo, portanto, seu efeito mediado na explicação do baixo peso ao nascer.

\section{Tabela 1}

Descrição das características sociodemográficas, do pré-natal e obstétricas, e análise bivariada conforme o baixo peso ao nascer em mães adolescentes e adultas jovens, Nordeste, Brasil, 2011-2012.

\begin{tabular}{|c|c|c|c|c|c|}
\hline \multirow[t]{2}{*}{ Características sociodemográficas } & \multirow[t]{2}{*}{ N } & \multirow[t]{2}{*}{$\%$} & \multicolumn{2}{|c|}{ Peso ao nascer $<2500 \mathrm{~g}$} & \multirow[t]{2}{*}{$p$} \\
\hline & & & $\mathrm{n}$ & $\%$ & \\
\hline \multicolumn{6}{|l|}{ Procedência } \\
\hline Interior & 2398 & 79,7 & 215 & 9,0 & 0,005 \\
\hline Capital & 611 & 20,3 & 78 & 12,8 & \\
\hline \multicolumn{6}{|l|}{ Situação conjugal } \\
\hline Sem companheiro & 674 & 22,4 & 63 & 9,3 & 0,691 \\
\hline Com companheiro & 2332 & 77,6 & 230 & 9,9 & \\
\hline \multicolumn{6}{|l|}{ Idade (anos) } \\
\hline $10-19$ & 1317 & 43,8 & 157 & 11,9 & $<0,001$ \\
\hline $20-24$ & 1692 & 56,2 & 136 & 8,0 & \\
\hline \multicolumn{6}{|c|}{ Adequação da escolaridade com a idade } \\
\hline Inadequada & 1806 & 60,9 & 181 & 10,0 & 0,444 \\
\hline Adequada & 1156 & 39,1 & 106 & 9,2 & \\
\hline \multicolumn{6}{|l|}{ Trabalho Remunerado } \\
\hline Sim & 500 & 16,6 & 41 & 8,2 & 0,204 \\
\hline Não & 2509 & 83,4 & 252 & 10,0 & \\
\hline \multicolumn{6}{|l|}{ Cor da pele } \\
\hline Preta & 231 & 7,7 & 24 & 10,4 & 0,546 \\
\hline Parda & 2208 & 73,4 & 208 & 9,4 & \\
\hline Branca & 473 & 15,7 & 52 & 11,0 & \\
\hline
\end{tabular}


Descrição das características sociodemográficas, do pré-natal e obstétricas, e análise bivariada conforme o baixo peso ao nascer em mães adolescentes e adultas jovens, Nordeste, Brasil, 2011-2012.

\begin{tabular}{|c|c|c|c|c|c|}
\hline \multirow[t]{2}{*}{ Características do pré-natal } & \multirow[t]{2}{*}{$\mathbf{N}$} & \multirow[t]{2}{*}{$\%$} & \multicolumn{2}{|c|}{ Peso ao nascer $<2500 \mathrm{~g}$} & \multirow[t]{2}{*}{$p$} \\
\hline & & & $\mathrm{n}$ & $\%$ & \\
\hline \multicolumn{6}{|c|}{ Número de consultas no pré-natal } \\
\hline$<6$ & 1007 & 35,3 & 153 & 15,2 & $<0,001$ \\
\hline$\geq 6$ & 1848 & 64,7 & 127 & 6,9 & \\
\hline \multicolumn{6}{|l|}{ Primigesta } \\
\hline Sim & 1743 & 57,9 & 193 & 11,1 & 0,004 \\
\hline Não & 1266 & 42,1 & 100 & 7,9 & \\
\hline \multicolumn{6}{|l|}{ Fumo durante a gestação } \\
\hline Sim & 169 & 5,6 & 21 & 12,4 & 0,225 \\
\hline Não & 2840 & 94,4 & 272 & 9,6 & \\
\hline \multicolumn{6}{|c|}{ Consumo de bebida alcoólica na gestação } \\
\hline Sim & 462 & 15,4 & 53 & 11,5 & 0,177 \\
\hline Não & 2541 & 84,6 & 240 & 9,4 & \\
\hline \multicolumn{6}{|c|}{ Acompanhada pelo mesmo profissional no pré-natal } \\
\hline Sim & 2667 & 90,7 & 249 & 9,3 & 0,136 \\
\hline Não & 272 & 9,3 & 33 & 12,1 & \\
\hline
\end{tabular}

\section{Características obstétricas}

\begin{tabular}{|c|c|c|c|c|c|}
\hline \multicolumn{6}{|c|}{ Financiamento para o parto } \\
\hline Público & 2870 & 95,4 & 283 & 9,9 & 0,300 \\
\hline Privado & 139 & 4,6 & 10 & 7,2 & \\
\hline \multicolumn{6}{|c|}{ Idade gestacional (semanas) } \\
\hline$<37$ & 321 & 10,9 & 166 & 51,7 & $<0,001$ \\
\hline$\geq 37$ & 2611 & 89,1 & 109 & 4,2 & \\
\hline \multicolumn{6}{|c|}{ Tipo de parto } \\
\hline Normal & 1774 & 59,1 & 182 & 10,3 & 0,228 \\
\hline Cesariana & 1231 & 40,9 & 110 & 8,9 & \\
\hline
\end{tabular}


Tabela 2

Resultados das regressões logísticas, distal, intermediário e proximal, tendo como variável resposta baixo peso ao nascer, Nordeste, Brasil, 2011-2012.

\begin{tabular}{|c|c|c|c|c|c|c|c|c|c|c|c|c|}
\hline \multirow[t]{2}{*}{ Modelo distal } & \multicolumn{4}{|c|}{ Modelo I } & \multicolumn{4}{|c|}{ Modelo II } & \multicolumn{4}{|c|}{ Modelo III } \\
\hline & OR & & $5 \%$ & $p$ & OR & & $5 \%$ & $p$ & OR & & $5 \%$ & $p$ \\
\hline Procedência & & & & 0,003 & & & & 0,014 & & & & 0,304 \\
\hline Interior & 0,6 & 0,4 & 0,8 & & 0,6 & 0,5 & 0,9 & & 0,8 & 0,5 & 1,1 & \\
\hline Capital & 1,0 & - & - & & 1,0 & - & - & & 1,0 & - & - & \\
\hline Idade (anos) & & & & $<0,001$ & & & & 0,073 & & & & 0,231 \\
\hline $10-19$ & 1,5 & 1,1 & 1,9 & & 1,2 & 1,0 & 1,6 & & 1,2 & 0,9 & 1,6 & \\
\hline $20-24$ & 1,0 & - & - & & 1,0 & - & - & & 1,0 & - & - & \\
\hline \multicolumn{13}{|l|}{ Modelo intermediário } \\
\hline Número de consultas & & & & - & & & & $<0,001$ & & & & $<0,001$ \\
\hline \multicolumn{13}{|l|}{ no pré-natal } \\
\hline$<6$ & - & - & - & & 2,4 & 1,8 & 3,1 & & 1,7 & 1,2 & 2,3 & \\
\hline$\geq 6$ & - & - & - & & 1,0 & - & - & & 1,0 & - & - & \\
\hline Primigesta & & & & - & & & & 0,001 & & & & 0,0005 \\
\hline Sim & - & - & - & & 1,6 & 1,2 & 2,1 & & 1,5 & 1,1 & 2,2 & \\
\hline Não & - & - & - & & 1,0 & - & - & & 1,0 & - & - & \\
\hline \multicolumn{13}{|l|}{ Modelo proximal } \\
\hline Idade gestacional (semanas) & & & & - & & & & - & & & & $<0,001$ \\
\hline$<37$ & - & - & - & & - & - & - & & 21,8 & 16,1 & 29,6 & \\
\hline$\geq 37$ & - & - & - & & - & - & - & & 1,0 & - & - & \\
\hline
\end{tabular}

\section{Discussão}

A dimensão continental do Brasil e suas grandes desigualdades sociais instigam e tornam relevante a investigação de fatores de risco para os desfechos da gestação, especialmente nos grupos populacionais mais expostos. Os resultados obtidos nesse estudo, que trata de amostra representativa de mães adolescentes e adultas jovens da Região Nordeste do Brasil e seus $\mathrm{RN}$ ratificam a contribuição de características do pré-natal e do parto na determinação do BPN entre conceptos desse grupo populacional.

A proporção de BPN encontrada nesse estudo $(11,9 \%)$ e $(8,0 \%)$ para adolescentes e adultas jovens, respectivamente, supera resultados de pesquisas realizados em Campinas (9,7\%) e no Rio Grande do Norte $(8,4 \%)$ entre adolescentes. Entretanto, outros estudos apontam valores superiores: no Maranhão (19,9\%) entre adolescentes; no Rio de Janeiro, $12,8 \%$, para adolescentes e $9,1 \%$, para adultas jovens; em Minas Gerais (Montes Claros), verificouse que a incidência de BPN entre filhos de adolescentes com idade até 15 anos foi de $35,2 \%, 11,7 \%$ naquelas de 15 a 19 anos e de $7,8 \%$ entre adultas.1,9,16,17,18 Esses achados reforçam a importância do acompanhamento assíduo desse grupo de gestantes pelos serviços de saúde, considerando o consenso da literatura que aponta o pré-natal adequado como fator de redução da vulnerabilidade e dos riscos atribuídos à idade cronológica entre gestantes adolescentes. ${ }^{11,12}$

No presente estudo, foi verificada maior prevalência de BPN, com significância estatística no grupo de puérperas que realizaram menos de seis consultas de pré-natal. A assistência inadequada do pré-natal tem sido indicada como importante fator de risco para desfechos adversos da gravidez, em especial no tocante ao BPN. No entanto, pesquisadores chamam a atenção que esse resultado pode refletir dificuldade de acesso a esse cuidado, principalmente em locais mais carentes, sendo um espelho da exclusão social. ${ }^{19} \mathrm{Em}$ pesquisa realizada no Novo México, mães residentes em áreas de baixa renda realizaram menos consultas de pré-natal e iniciaram mais tardiamente em relação às demais, corroborando com os resultados dessa pesquisa. 20 
Os benefícios da assistência pré-natal já foram identificados em estudos realizados em diferentes contextos, regiões e países. 21,22 Em estudo realizado no Irã, pesquisadores observaram que o peso do recém-nascido de mães que realizaram pré-natal adequado foi significativamente maior. ${ }^{23}$ Nos Estados Unidos, pesquisas apontaram que a prevalência de baixo peso ao nascer foi menor nos $\mathrm{RN}$ de gestantes que receberam assistência pré-natal precocemente, a partir do primeiro trimestre, em comparação às que iniciaram no segundo ou terceiro trimestre. ${ }^{24}$

No Brasil, a maioria dos prematuros nasce com idade gestacional entre 34 e 36 semanas e peso superior a $2500 \mathrm{~g}$, entretanto, apesar do aumento da frequência de prematuridade, a prevalência de baixo peso ao nascer tem se estabilizado no patamar de $8 \%$, desde o ano 2000 , possivelmente pela redução da frequência do retardo de crescimento intrauterino. 25

No presente estudo, a prematuridade esteve fortemente associada ao BPN, sendo que as crianças nascidas antes de 37 semanas tiveram 21 vezes mais chance de pesar menos de $2500 \mathrm{~g}$ em relação àquelas de maior idade gestacional, corroborando com a consensual literatura. Considerando-se que 51,7\% dos bebês prematuros nasceram com menos de 2500 $\mathrm{g}$, é possível que a maior frequência de $\mathrm{RN}$ com baixo peso entre os filhos de mães que realizaram menor número de consultas no pré-natal se deva ao fato de não terem tido oportunidade de completar o número preconizado de consultas, pela interrupção precoce da gestação.

Estudos realizados nas cidades de São Paulo e de Campinas verificaram entre prematuros um risco de 20 e 35 vezes maior para o baixo peso ao nascer, respectivamente.9,26 Possivelmente, para esse alto risco, o diferencial se deva ao tamanho da população estudada e mesmo à sua composição, ou seja, o nível de prematuridade entre esses recém-nascidos.

Pesquisa realizada em Aracaju, com nascidos

\section{Referências}

1. Santos GHN, Martins, MG, Sousa MS. Gravidez na adolescência e fatores associados com baixo peso ao nascer. Rev Bras Ginecol Obstet. 2008; 30: 224-31.

2. Cesar JA, Mendoza-Sassi RA, Gonzales-Chica DA, Mano PS, Goulart-Filha SM. Características sociodemográficas e de assistência à gestação e ao parto no extremo sul do Brasil. Cad Saúde Pública. 2011; 27: 985-94.

3. Brasil. Ministério da Saúde. Secretaria de Atenção à Saúde. Homepage do Banco de Dados do Sistema Único de Saúde - DATASUS. [acesso em 14 abr. 2014]. Disponível em: www.datasus.gov.br vivos de baixo peso revelou que a idade gestacional da maioria das crianças estudadas foi inferior a 37 semanas, provavelmente, uma consequência do menor número de consultas pré-natal realizado pelas gestantes. ${ }^{21}$ No Maranhão, verificou-se que os fatores de risco para prematuridade foram mais evidentes nas adolescentes e entre aquelas que não frequentaram o programa de pré-natal. 27

As análises apontaram que o risco social (baixa adesão ao pré-natal, localização geográfica, entre outros), associado ao risco biológico (idade, primigestação e prematuridade) possam ter interferido na prevalência de baixo peso entre conceptos de mães adolescentes da Região Nordeste do Brasil. Não obstante, chama a atenção o elevado percentual de cesarianas e da prematuridade que tem impacto decisivo na saúde materno infantil da região, além do considerável número de mulheres que afirmaram ter fumado e consumido bebida alcoólica na gestação. Este é um fato preocupante, justamente pelo efeito nocivo do uso dessas substâncias durante o período gestacional, bem como por se tratar de uma população jovem. 28,29

O estudo demonstrou alta frequência do baixo peso ao nascer entre recém-nascidos de adolescentes e adultas jovens, tendo como principais fatores associados o número insuficiente de consultas pré-natal, a primigestação e a prematuridade.

Os determinantes do BPN variam em efeitos e magnitude entre diferentes populações. Assim, o atual estudo, além de reforçar achados da literatura, como a necessidade de adequação e ampliação da assistência pré-natal, contribui para levantar indicadores que possibilitam mapear e dimensionar o fenômeno, em uma grande e importante região do país, considerando a representatividade do objeto de estudo. Estes indicadores são decisivos para gestores, na implementação de políticas e programas direcionados à população materno infantil, especialmente nos grupos mais vulneráveis.

4. Guimarães AMDN, Bettiol $\mathrm{H}$, Souza L, Gurgel RQ, Almeida MLD, Ribeiro ERO et al. Gravidez na adolescência é fator de risco para o baixo peso ao nascer? Rev Saúde Pública. 2013; 47: 11-9.

5. Viana KJ, Taddei JAAC, Cocetti M, Warkentin S. Peso ao nascer de crianças brasileiras menores de dois anos. Cad Saúde Pública. 2013; 29: 349-56.

6. Kumar SG, Kumar HNH, Jayaram S, Kotian MS. Determinants of Low Birth Weight: A Case Control Study in a District Hospital in Karnataka. Indian J Pediatr. 2010; 77: 87-9. 
7. Simões VM, da Silva AA, Bettiol H, Lamy-Filho F, Tonial SR, Mochel EG. Características da gravidez na adolescência em São Luís, Maranhão. Rev Saúde Publica. 2003; 37: 55965.

8. Silva CF, Leite AJ, Almeida NM, Gondim RC. Fatores de risco para mortalidade infantil em município do Nordeste do Brasil: linkage entre bancos de dados de nascidos vivos e óbitos infantis - 2000 a 2002. Rev Bras Epidemiol. 2006; 9: 69-80.

9. Carniel EF, Zanolli ML, Antônio MARGMA, Morcillo AM. Determinantes do baixo peso ao nascer a partir das Declarações de Nascidos Vivos. Rev Bras Epidemiol. 2008; 11: 169-79.

10. Silva AAM, Silva LM, Barbieri MA, Bettiol H, Carvalho LM, Ribeiro VS, et al. O paradoxo epidemiológico do baixo peso ao nascer no Brasil. Rev Saúde Publica. 2010; 44: 767-75.

11. Smith GCS, Pell JP, Walsh D. Pregnancy complications and maternal risk of ischaemic heart disease: a retrospective cohort study of 129,290 births. Lancet. 2001; 357: 2002-06

12. Raatikainen K, Heiskanen N, Heinonen S. Marriage still protects pregnancy. BJOG. 2005; 112 (10): 1411-6.

13. Leal MC, Silva AAM, Dias MAB, Gama SGN, Rattner D, Moreira ME et al. Birth in Brazil: national survey into labour and birth. Reproductive Health 2012; 9:15

14. Vasconcellos MTL, Silva PLN, Esteves-Pereira AP, Schilithz AOC, Souza Junior PRB, Szwarcwald CL. Desenho da amostra Nascer no Brasil: Pesquisa Nacional sobre Parto e Nascimento. Cad Saúde Pública. 2014; 30 (Supl.): 49-58.

15. Esteves-Pereira AP, Leal MC, Gama SGN, Domingues RMSM, Schilithz AOC, Bastos MH. Determinação da idade gestacional com base em informações do estudo Nascer no Brasil. Cad Saúde Pública. 2014; 30: 59-70.

16. Azevedo GD, Freitas Júnior RAO, Freitas AKMSO, Araújo ACPF, Soares EMM, Maranhão TMO. Efeito da idade materna sobre os resultados perinatais. Rev Bras Ginecol Obstet. 2002; 24: 181-5.

17. Gama SGN, Szwarcwald CL, Leal MC. A gravidez na adolescência como fator de risco para o baixo peso ao nascer no Município do Rio de Janeiro, 1996 a 1998. Rev Saúde Pública. 2001; 35: 74-80.

18. Goldenberg P, Figueiredo MCT, Silva RS. Gravidez na adolescência, pré-natal e resultados perinatais em Montes Claros, Minas Gerais, Brasil. Cad Saúde Pública. 2005; 21 1077-86.

Recebido em 2 de abril de 2014

Versão final apresentada em 17 de junho de 2014

Aprovado em 30 de junho de 2014
19. Schoeps D, Furquim de Almeida M, Alencar GP, França Jr I, Novaes HM, Franco de Siqueira AA, Campbell O, Rodrigues LC. Fatores de risco para mortalidade neonatal precoce. Rev Saúde Publica. 2007; 41: 1013-22.

20. Schillaci MA, Waitzkin H, Carson EA, Romain SJ. Prenatal care utilization for mothers from low-income areas of New Mexico, 1989-1999. PLoS ONE. 2010; 5: 3-6.

21. Ribeiro ERO, Guimarães AMDN, Bettiol H, Lima DDF, Almeida ML, Souza L, Silva AAM, Gurgel RQ. Risk factors for inadequate prenatal care use in the metropolitan area of Aracaju, Northeast Brazil. BMC Pregnancy Childbirth. 2009; 9:31.

22. Silveira MF, Victora CG, Barros AJD, Santos IS, Matijasevich A, Barros FC. Determinants of preterm birth: Pelotas, Rio Grande do Sul State, Brazil, 2004 birth cohort. Cad Saúde Pública. 2010; 26: 185-94.

23. Ashraf-Ganjoei T, Mirzaei F, Anari-Dokht F. Relationship between prenatal care and the outcome of pregnancy in low-risk pregnancies. Open J Obstet Gynecol. 2011; 1: 10912.

24. Hueston JH, Gregory EG, Davis L, Sturgill V. Delayed prenatal care and the risk of low birth weight delivery. J Community Health. 2003; 28: 199-208.

25. Victora CG, Aquino EM, Leal MC, Monteiro CA, Barros FC, Szwarcwald CL. Saúde de mães e crianças no Brasil: progressos e desafios. Lancet. 2011; 377: 1863-76.

26. Monteiro CA, Benício MHA, Ortiz LP. Tendência secular do peso ao nascer na cidade de São Paulo (1976-1998). Rev Saúde Pública. 2000; 34: 26-40.

27. Aragão VM, Silva AA, Aragão LF, Barbieri MA, Bettiol H, Coimbra LC, Ribeiro VS. Risk factors for preterm births in Sao Luís, Maranhão, Brazil. Cad Saúde Pública. 2004; 20 : 57-63.

28. Bernstein IM, Mongeon JA, Badger GJ, Solomon L, Heil SH, Higgins ST. Maternal smoking and its association with birth weight. Obstet Gynecol. 2005; 106: 986-91.

29. Zhang L, González-Chica DA, Cesar JA, Mendoza-Sassi RA, Beskow B, Larentis N, Blosfeld T. Tabagismo materno durante a gestação e medidas antropométricas do recémnascido: um estudo de base populacional no extremo sul do Brasil. Cad. Saúde Pública. 2011; 27: 1768-1776. 